\title{
Neuroradiology
}

\section{Black Bone MRI with 3D reconstruction for the Detection of Skull Fractures in Children with Suspected Abusive Head Trauma

\author{
--Manuscript Draft--
}

\begin{tabular}{|c|c|}
\hline Manuscript Number: & NRAD-D-18-00432 \\
\hline Full Title: & $\begin{array}{l}\text { Black Bone MRI with 3D reconstruction for the Detection of Skull Fractures in Children } \\
\text { with Suspected Abusive Head Trauma }\end{array}$ \\
\hline Section/Category: & Paediatric Neuroradiology \\
\hline Corresponding Author: & $\begin{array}{l}\text { Stephen F. Kralik } \\
\text { Indiana University School of Medicine } \\
\text { Indianapolis, UNITED STATES }\end{array}$ \\
\hline \multicolumn{2}{|l|}{$\begin{array}{l}\text { Corresponding Author's Secondary } \\
\text { Institution: }\end{array}$} \\
\hline Author Comments: & $\begin{array}{l}\text { Dear Neuroradiology Editors, } \\
\text { I am pleased to submit for review on behalf of myself and my co-authors the following } \\
\text { original research paper titled "Black Bone MRI with 3D reconstruction for the Detection } \\
\text { of Skull Fractures in Children with Suspected Abusive Head Trauma." } \\
\text { Black bone MRI sequences are newer MRI sequences which have been developed for } \\
\text { imaging of the bone with MRI. The purpose for this original research was to compare a } \\
\text { black bone MRI sequence to head CT for detection of skull fractures in pediatric } \\
\text { patients with possible abusive head trauma. } \\
\text { This manuscript has not been previously published or submitted concurrently to any } \\
\text { other journal. The co-authors have read and approved this submission to this journal. } \\
\text { Sincerely, } \\
\text { Stephen F. Kralik M.D. } \\
\text { Assistant Professor of Radiology } \\
\text { Department of Radiology, Texas Children's Hospital } \\
\text { Mark A. Wallace Tower, } 4 \text { th Floor } \\
6701 \text { Fannin St, Suite } 470 \\
\text { Houston, TX, } 77030\end{array}$ \\
\hline First Author: & Stephen F. Kralik \\
\hline \multicolumn{2}{|l|}{ First Author Secondary Information: } \\
\hline \multirow[t]{7}{*}{ Order of Authors: } & Stephen F. Kralik \\
\hline & Nucharin Supakul, MD \\
\hline & Isaac C Wu, MD \\
\hline & Gaspar Delso, PhD \\
\hline & Rupa Radhakrishnan, MD \\
\hline & Chang Y. Ho, MD \\
\hline & Karen A. Eley, MD, FRCR, DPhil \\
\hline
\end{tabular}

Order of Authors Secondary Information: 


\begin{tabular}{|c|c|}
\hline Funding Information: & \\
\hline Abstract: & 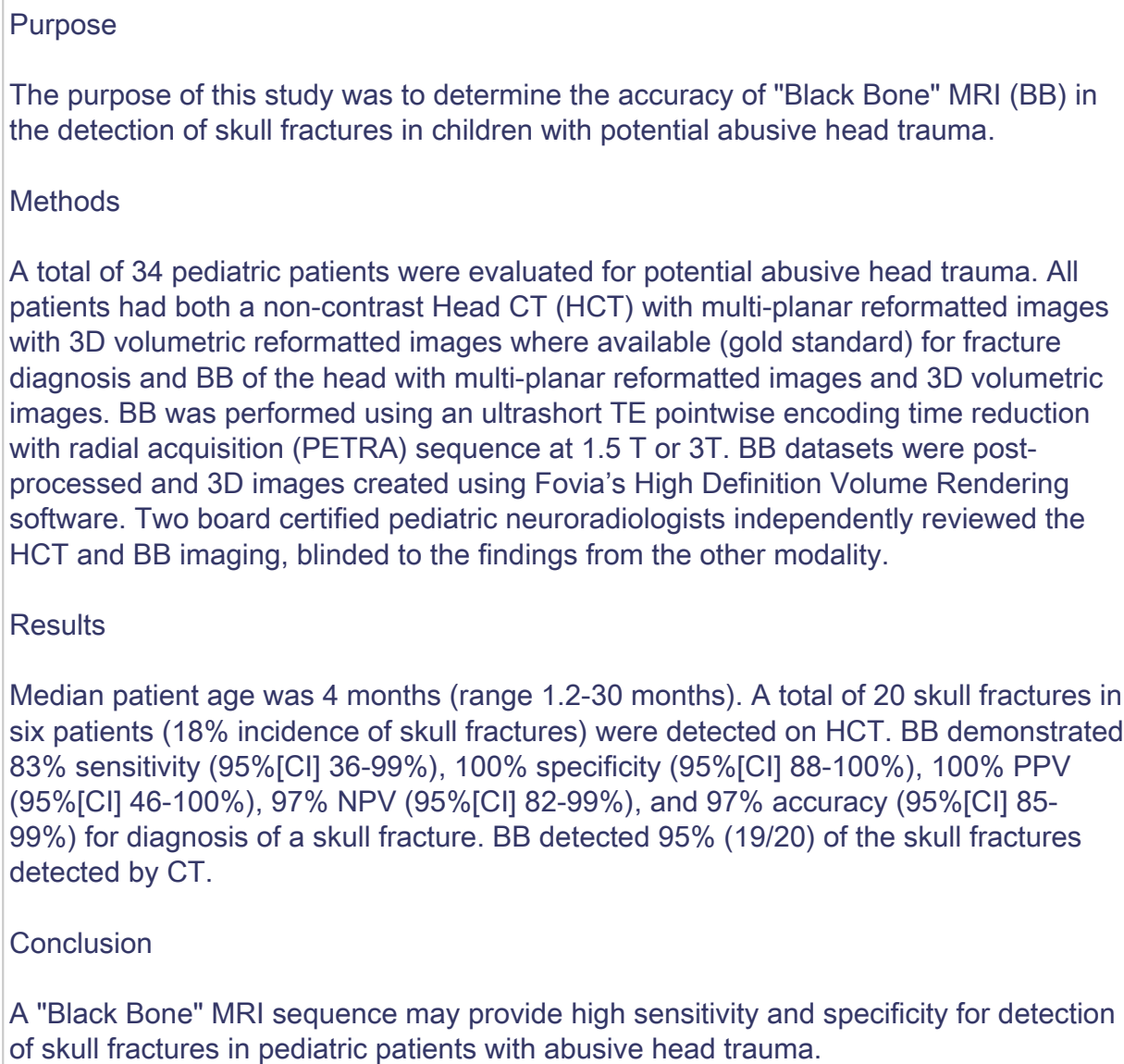 \\
\hline
\end{tabular}




\title{
Black Bone MRI with 3D reconstruction for the Detection of Skull Fractures in Children with Suspected Abusive Head Trauma
}

\begin{abstract}
Purpose

The purpose of this study was to determine the accuracy of "Black Bone" MRI (BB) in the detection of skull fractures in children with potential abusive head trauma.
\end{abstract}

\section{Methods}

A total of 34 pediatric patients were evaluated for potential abusive head trauma. All patients had both a non-contrast Head CT (HCT) with multi-planar reformatted images with 3D volumetric reformatted images where available (gold standard) for fracture diagnosis and BB of the head with multi-planar reformatted images and 3D volumetric images. BB was performed using an ultrashort TE pointwise encoding time reduction with radial acquisition (PETRA) sequence at 1.5 $\mathrm{T}$ or 3T. BB datasets were post-processed and 3D images created using Fovia's High Definition Volume Rendering software. Two board certified pediatric neuroradiologists independently reviewed the $\mathrm{HCT}$ and $\mathrm{BB}$ imaging, blinded to the findings from the other modality.

\section{Results}

Median patient age was 4 months (range 1.2-30 months). A total of 20 skull fractures in six patients (18\% incidence of skull fractures) were detected on HCT. BB demonstrated $83 \%$ sensitivity (95\%[CI] 36-99\%), 100\% specificity (95\%[CI] 88-100\%), 100\% PPV (95\%[CI] 46100\%), 97\% NPV (95\%[CI] 82-99\%), and 97\% accuracy (95\%[CI] 85-99\%) for diagnosis of a skull fracture. BB detected 95\% (19/20) of the skull fractures detected by CT. 


\section{Conclusion}

A "Black Bone" MRI sequence may provide high sensitivity and specificity for detection of skull fractures in pediatric patients with abusive head trauma.

Abbreviations: UTE: ultrashort TE; PETRA: pointwise encoding time reduction with radial acquisition; HCT: head CT; AHT: abusive head trauma

\section{Introduction}

The estimated incidence of abusive head trauma (AHT) is 39.8 per 100,000 children younger than 1 year of age.[1] The outcomes of these victims are worse than those of accidental traumatic brain injury, including higher rates of mortality and neurological impairments.[2-5] The diagnosis of AHT requires a multidisciplinary approach, including radiology and neuroimaging, usually with CT and/or MRI. There are advantages and disadvantages for both CT and MR imaging for the detection of AHT. A noncontrast head CT (HCT) is usually the initial imaging study in suspected AHT due to its high sensitivity for the detection of acute hemorrhage and fracture, the high level of accessibility from the emergency department, and it can be performed quickly and safely without the need for special monitoring equipment.[6,7] The disadvantages of CT include ionizing radiation, particularly in children, and the reduced sensitivity in detecting microhemorrhages, axonal injury, and acute ischemia compared with MR imaging.[8]

The term "Black Bone" was first reported in the literature by Eley et al in 2012 to describe the MRI technique of enhancing the bone - soft-tissue interface by minimizing the 
signal returned from all tissues by utilizing a short TE/TR and low flip-angle. $[9,10]$ Comparable BB imaging can be achieved utilizing ultrashort TE (UTE) using pointwise encoding time reduction with radial acquisition (PETRA; Siemens, Erlangen Germany) which is a relatively new MRI sequence that utilizes TE times in the microseconds allowing for tissue such as bone to have a dark signal intensity. The PETRA sequence is a 3D MRI sequence that can obtain isotropic resolution with $1 \mathrm{~mm}$ slice thickness of the head in a short time period ( 4 minutes).

$\mathrm{BB}$ has been shown to be useful in a range of craniofacial pathologies, including in the diagnosis of craniosynostosis in infants.[10-12] One of the key benefits of the technique is the ability to create 3D reconstructed images of the craniofacial skeleton and 3D printed anatomical models. $[11,13,14]$

3D reconstructed imaging has been shown to increase the sensitivity and specificity of HCT in the detection of skull fractures.[15] In a recent study BB was found to be less sensitive and specific compared to HCT for detection of skull fracture in pediatric trauma patients; however, to our knowledge $\mathrm{BB}$ with 3D reconstruction has not previously been evaluated in this patient group.[16] The purpose of this study was to determine the accuracy of multi-planar BB with 3D reconstruction in the detection of skull fractures in children with potential AHT.

\section{Materials and Methods}

Following institutional review board approval, a retrospective study was performed from 2017-2018 on 34 pediatric patients (age < 18 years) who had presented acutely to the emergency department and undergone both HCT and BB for potential AHT. Patients were included if they had undergone a non-contrast HCT within the preceding five days, and MR imaging of the head 
had been requested to further evaluate the patient for potential AHT. HCT was performed either at the referring institution or upon initial presentation to our institution.

The MRI brain protocol was performed with or without an MRI compatible immobilizer (MedVac Infant Immobilizer; CFI Medical, Fenton, Michigan). At our institution, an immobilizer is routinely used for infants younger than 3 months of age and general anesthesia is used for children between the ages of 3 months and 7 years. MR imaging was performed with 1.5T or 3T scanners (Avanto, or Skyra; Siemens, Erlangen, Germany) using a 20 channel head coil. The UTE PETRA MRI was performed at $1.5 \mathrm{~T}$ and $3 \mathrm{~T}$ with TR/TE 3.32/0.07 ms, matrix $224 \times 224$, field of view $25 \times 25 \mathrm{~cm}$, slice thickness $1 \mathrm{~mm}$. MR imaging technologists were instructed to repeat the PETRA sequence only once if there were severe motion artifacts. Multiplanar reformatted images of the PETRA images were performed in the coronal and sagittal planes with $1 \mathrm{~mm}$ slice thickness.

3D volume rendering of the craniofacial skeleton was completed by one author (K.E.) who was blinded to the diagnosis and patient demographics. Following automated removal of the outer skin envelope, the DICOM datasets were rendered using Fovia's High Definition Volume Rendering ${ }^{\oplus}$ software (Fovia Inc., Palo Alto, CA, USA).[11,13] Standardized 3D imaging movie clips with 360 degree right-left rotating and head-feet images were created.

A non-contrast Head CT (HCT) with multi-planar reformatted images with 3D volumetric reformatted images (where available) were used as the gold standard for skull fracture diagnosis. All HCTs performed at our institution were performed on a 256 multidetector CT scanner (Philips Brilliance iCT, Best, Netherlands) with the following parameters: $100 \mathrm{kV} ; 196 \mathrm{~mA}, 1 \mathrm{~mm}$ slice thickness. Axial bone filter images were reconstructed at $2.5 \mathrm{~mm}$ 
slice thickness. Multi-planar HCT images were performed with $\leq 2 \mathrm{~mm}$ slice thickness and volume rendered 3D reconstructed images consisted of 360 degree right-left rotating and headfeet images were created on Philips IntelliSpace Portal 8.0 (Philips Healthcare, Best, Netherlands). A total of 25 patients were imaged at our institution with this HCT protocol. HCTs were performed in the remaining 9 patients at referring institutions with the requirement for patient inclusion that all HCTs must have axial slice thickness $\leq 2.5 \mathrm{~mm}$ and coronal and/or sagittal reformatted images. 3D reconstructions were available in five of the nine patients imaged at referring institutions while the remaining four patients did not have 3D reconstructions nor source data available to permit HCT 3D reconstruction.

Two board-certified fellowship-trained pediatric neuroradiologists (N.S., I.W.) with certificates of added qualification in neuroradiology, with three and nine years of experience respectively, independently reviewed the axial, multi-planar, and 3D HCT and axial and multiplanar BB images on a Fuji PACS workstation. 3D-BB images were reviewed on a separate computer using a video player (Windows Media Player, Windows 7 enterprise, Microsoft Corporation, Richmond, WA, USA). Images were evaluated for diagnosis of a skull fracture (yes/no), and description of fracture including location, number of fractures, and fracture depression (yes/no). HCT and BB images were reviewed separately with a 1-month interval to avoid memory bias. The pediatric neuroradiologists were aware that the clinical indication for all cases was for evaluation of potential AHT but were otherwise blinded to the final clinical interpretation of child abuse, and additional clinical and radiologic information of the patient, including skeletal survey results. Discrepancies between the reviewers were resolved by a third reviewer (S.K.) with certificate of added qualification in neuroradiology and seven years of experience. 
Inter-reader concordance was calculated using kappa $(\mathrm{K})$ value with $\mathrm{K}$ values $<0$ considered no agreement; $0-0.20$, as slight agreement; $0.21-0.40$, as fair agreement; $0.41-0.60$, as moderate agreement; 0.61-0.80, as substantial agreement; and 0.81-1, as almost perfect agreement.[17] Sensitivity, specificity, positive predictive value (PPV), negative predictive value (NPV) and accuracy for consensus interpretation for BB diagnosis of calvarial fracture per patient, rather than by number of fractures, were calculated compared with consensus HCT as the gold standard using a 2 x 2 contingency table. If any one of multiple fracture was missed in one patient, that patient would be considered a missed diagnosis. Subgroup sensitivity, specificity, PPV, NPV and accuracy was calculated for patients who had 3D CT reconstructions (30 patients), $1.5 \mathrm{~T}$ vs $3 \mathrm{~T}$ and patients imaged without sedation versus with sedation. The $\mathrm{Z}$ ratio was used to assess the significance of the proportions of false negative patients in these subgroups and $\mathrm{P}<0.05$ was considered statistically significant. The statistics were performed by using a commercially available statistical software (MedCalc Software, Version 14.12.0, Mariakerke, Belgium).

\section{Results}

Median patient age was 4 months (mean 5.2 months; range 1.2-30 months) and the male:female ratio was 14:20. General anesthesia was utilized in 47\% (16/34) of patients. Imaging was performed at $1.5 \mathrm{~T}$ in $32 \%(11 / 34)$ and $3 \mathrm{~T}$ in $68 \%(23 / 34)$. BB imaging was performed at a median of 1 day (range 0-4 days) following HCT. There were no BB imaging datasets that were considered non-diagnostic or required repeat imaging.

The incidence of skull fracture by HCT was $18 \%$ (6/34 patients). A total of 20 skull 
fractures were identified by HCT in the six patients. Three fractures were depressed or displaced. All fractures occurred in the parietal or occipital bones. Concordance for CT diagnosis of fracture by the reviewers was $0.89(95 \%[\mathrm{CI}] 0.68-1.0)$ indicating almost perfect agreement.

BB results are shown in Table 1. BB demonstrated 83\% sensitivity (95\%[CI] 36-99\%), $100 \%$ specificity $(95 \%[\mathrm{CI}] 88-100 \%), 100 \% \mathrm{PPV}(95 \%[\mathrm{CI}] 46-100 \%), 97 \%$ NPV (95\%[CI] $82-$ 99\%), and $97 \%$ accuracy (95\%[CI] 85-99\%) for diagnosis of a skull fracture. Comparisons of sensitivity, specificity, PPV, NPV and accuracy for BB performed at $1.5 \mathrm{~T}$ vs $3 \mathrm{~T}$ and sedation vs no sedation are shown in Table 1. For the subgroup of 30 patients who all had 3D HCT reconstructions, there was $80 \%$ sensitivity, $100 \%$ specificity, $100 \% \mathrm{PPV}, 96 \% \mathrm{NPV}$, and $97 \%$ accuracy. There was decreased sensitivity among patients imaged at 3T versus $1.5 \mathrm{~T}$ ( $75 \%$ vs $100 \%)$ and among patients imaged without sedation versus patients imaged with sedation (50\% vs $100 \%)$, however, neither were statistically significant ( $\mathrm{P}=0.48$ and $\mathrm{P}=0.37$ respectively). $\mathrm{BB}$ detected 95\% (19/20) of the fractures identified on CT. Concordance for BB diagnosis of fracture by the reviewers was $0.89(95 \%[\mathrm{CI}] 0.68-1.0)$ indicating perfect agreement. Representative HCT and MRI images are shown in Figures 1-3. The single false negative case was a 9-month old patient who was imaged without sedation at 3T (Figure 3). This fracture was missed by one reviewer on HCT representing the lone HCT discordant case.

\section{Discussion}

In this study, BB using UTE PETRA with multi-planar and 3D reconstruction achieved a sensitivity of $83 \%$ and specificity of $100 \%$ compared to HCT as the gold standard. These results are higher than those of Dremman et al who reported a sensitivity of $67 \%$ and specificity of $88 \%$ 
for BB compared to HCT.[16] There are a few important differences between our study and that of Dremman et al that may account for this difference. First, although both studies used isotropic $1 \mathrm{~mm}$ slice thickness to perform multi-planar reformatted images, we additionally performed post processing of the BB PETRA datasets to create 3D reconstructed bone imaging. These volume rendered images of the calvarium improved fracture diagnosis, similar to research by Orman et al who demonstrated increased sensitivity and specificity in fracture detection in children with HCT using 3D reconstruction.[15] Dremman et al notably reported that the misinterpretations of $\mathrm{BB}$ involved differentiation of fracture from a cranial suture and we suspect that the $3 \mathrm{D}$ reconstruction assists in more accurate differentiation.[16]

Accurate segmentation of the craniofacial skeleton remains challenging in young infants due to the thin calvarial bone. It is therefore necessary at present to utilize manual techniques to achieve the final segmentation results, which can be time intensive thus limiting translation into routine clinical practice. We incorporated an automated process to remove the outer soft tissue envelope which reduced the time required to 3D render the datasets in this study. Rendering of the facial skeleton was particularly challenging and we could have reduced the 3D rendering time to under 5 minutes if we had segmented the calvarium in isolation. Phase-wrap resulted in artificial defects occurring at the skull vertex on some of the $3 \mathrm{D}$ reconstructions. In three cases a higher frequency and bandwidth had been used with good 3D results; however, there was no discernible correlation between the most optimal 3D results and the imaging parameters nor patient age, which was a little surprising. While Fovia produces aesthetically pleasing results, the reliance on visual interrogation of the final result (where the most minor change within the transfer function can significantly alter the 3D rendering), may have impacted upon the diagnostic accuracy of fracture detection. The authors (K.E., G.D.) continue to investigate the 
optimal BB imaging parameters and 3D techniques with the aim of achieving a fully automated segmentation algorithm to permit incorporation into routine clinical practice. It is anticipated that refinement of imaging parameters in combination with a fully automated segmentation technique would further improve the diagnostic accuracy of 3D-BB in the detection of skull fractures.

Our study focused on patients with potential AHT with a mean age of 5.2 months, while Dremman et al evaluated all pediatric trauma patients, with a higher mean age (4.89 years) and wider age range (6 days to 15.5 years).[16] As younger patients have thinner calvarial bone thickness and unfused sutures, our cohort would represent a more challenging population to accurately detect fractures with MRI. Despite the younger mean age of our patient cohort we did not encounter any misinterpretations due to the cranial sutures. This may be the result of the added benefit of $3 \mathrm{D}$ reconstruction performed in our study, or the shorter TE achieved with the UTE PETRA sequence $(0.07 \mathrm{~ms}$ compared to $4.2 \mathrm{~ms})$ resulting in improved bone-soft tissue interface depiction.

Demonstration of BB imaging that is comparable to HCT for fracture detection would have value in allowing reduction in ionizing radiation. Currently, when the pretest probability of of head trauma is high enough to merit a HCT, the theoretical risk from ionizing radiation is a negligible consideration compared to the risk of missing intracranial injury. The lifetime cancer mortality risk from a single HCT in a one-year old child is estimated to be 0.07\%.[18] This translates to larger risk at the population level because head trauma in children 0 to 14 years of age accounts for nearly half a million emergency department visits in the United States annually. [16] Radiation exposure from HCT is a concern in children at risk for AHT due to their young age and exposure of the lens and thyroid during imaging. However, for MRI to be a viable alternative to HCT the BB technique (PETRA or equivalent) must consistently produce 
diagnostic quality imaging, and must be cost-effective. In young children even with short acquisition times MRI examination may necessitate sedation or general anesthesia to achieve good quality imaging. The PETRA sequence used in this study required only 4 minutes of imaging time which is equivalent to many standard MRI sequences. PETRA imaging was designed as an MRI sequence that has less noise production which may aid in imaging of young children.[19] In this study, we had no non-diagnostic studies and no studies requiring repeat imaging. Importantly however, $47 \%$ of patients were imaged under general anesthesia which may influence the reproducibly in acquiring diagnostic BB images. We did not attempt to perform MRI without sedation, in line with current routine clinical care at our institution. This factor may be important if there is expanded utilization of BB imaging for pediatric imaging. There was a decrease in sensitivity, although not statistically significant, between sedated and non-sedated children, and between patients imaged at $3 \mathrm{~T}$ versus 1.5T. Differences in diagnosis in sedated versus non-sedated patients may be attributable to motion artifact, or younger age as our non-sedated MRIs were primarily in the first 3 months of life. As UTE PETRA imaging utilizes radial $\mathrm{k}$-space sampling to shorten echo time, the sequence is sensitive to inherent hardwarebased gradient timing errors, eddy currents and main magnetic field inhomogeneities, however radial $\mathrm{k}$-space sampling is robust with respect to patient motion.[20]

Our study has a few important limitations that should be noted. First, this was a clinical feasibility study with a relatively small number of patients. In addition, there was a proportionately low number of fractures because the prevalence of fractures in our cohort was approximately $20 \%$, and fractures were limited to parietal and occipital bones. The results indicate potential value of "black bone" MRI in this patient population warranting further investigation potentially with longer recruitment time frame or multicenter trial. A potential 
limitation of the BB technique is fractures occurring at an air-bone interface such as the mastoid portion of the temporal bone or the paranasal sinuses, due to the overlapping pixel values of bone and air. Skull fractures are common in children with head trauma, and the majority are linear fractures.[15,16,21-23] Although an isolated linear skull fracture has low clinical significance, precise diagnosis of injuries in patients with potential AHT is important for medicolegal purposes and may still require HCTs.

Another potential limitation of the study may include a selection bias. All patients who had BB had preceding HCT and required MRI for further evaluation. This scenario may introduce factors that would be different from one in which MRI was the sole imaging modality used to detect AHT. In addition, our patient population may include AHT patients with more serious injury than patients who only had CT imaging to assess for AHT. However, our patient selection allowed us to compare $\mathrm{HCT}$ to $\mathrm{BB}$ MRI for fracture detection, as well as evaluate $\mathrm{BB}$ MRI in a population of patients whose calvarium is thin. This selection bias also resulted in a small number of patients who had HCT performed at the referring institution with different CT technique, including lack of 3D reconstruction in four patients. In order to reduce radiation dose, our institution does not repeat HCT if the referring institution HCT is considered diagnostic. Lastly, we chose to focus on fracture detection rather than evaluate additional intracranial trauma findings because previous reports have demonstrated superiority of MRI compared to CT in pediatric patients.[16,24]

Nevertheless, based on the results of our study, BB imaging with 3D reconstruction is a promising tool to detect calvarial fractures in young children with suspected abusive head trauma. Further refinement in technique and improvements in the processing pipeline of the BB 
images could facilitate its use in other conditions currently requiring CT imaging for assessment of bone detail.

\section{Conclusion}

Among pediatric patients referred for MRI to evaluate potential AHT, the BB demonstrated high sensitivity and specificity for detection of skull fracture compared to the gold standard HCT. Larger studies are necessary to confirm the potential for BB to replace HCT in the neuroimaging evaluation of patients with potential AHT.

\section{References:}

1. Niederkrotenthaler T, Xu L, Parks SE, SugermanDE. Descriptive factors of abusive head trauma in young children: United States, 2000-2009. Child Abuse Negl 2013;37:446-55

2. Duhaime AC, Christian C, Moss E, Seidl T. Long-term outcome in infants with the shaking-impact syndrome. Pediatr Neurosurg 1996 Jun;24(6): 292-298

3. Chevignard MP, Lind K. Long-term outcome of abusive head trauma. Pediatr Radiol 2014 Dec;44 Suppl 4:S548-S558

4. Reece RM, Sege R. Childhood head injuries: accidental or inflicted? Arch Pediatr Adolesc Med 2000 Jan;154(1):11-15

5. Sills MR, Libby AM, Orton HD. Prehospital and in-hospital mortality: a comparison of intentional and unintentional traumatic brain injuries in Colorado children. Arch Pediatr Adolesc Med 2005 Jul;159(7): 665-670

6. Jaspan T, Griffiths PD, McConachie NS, et al. Neuroimaging for non-accidental head injury in childhood: a proposed protocol. Clin Radiol 2003 Jan;58(1):44-53 
7. Ginde AA, Foianini A, Renner DM, Valley M, Camargo CA. Availability and quality of computed tomography and magnetic resonance imaging equipment in U.S. emergency departments. Acad Emerg Med 2008 Aug;15(8):780-783

8. Hedlund GL, Frasier LD. Neuroimaging of abusive head trauma. Forensic Sci Med Pathol 2009 Dec;5(4):280-90

9. Eley KA, McIntyre AG, Watt-Smith SR, Golding SJ. "Black Bone” MRI: a partial flip angle technique for radiation reduction in craniofacial imaging. Br J Radiol 2012; 85(1011): 272-8

10. Eley KA, Watt-Smith SR, Golding SJ. "Black Bone” MRI: a potential alternative to CT when imaging the head and neck: report of eight clinical cases and review of the Oxford experience. Br J Radiol 2012; 85(1019):1457-64

11. Eley KA, Watt-Smith SR, Sheerin F, Golding SJ. "Black Bone" MRI: a potential alternative to CT with three-dimensional reconstruction of the craniofacial skeleton in the diagnosis of craniosynostosis. Eur Radiol. 2014 Oct;24(10):2417-26.

12. Eley KA, Watt-Smith SR, Golding SJ. "Black Bone" MRI: a potential non-ionizing method for three-dimensional cephalometric analysis--a preliminary feasibility study. Dentomaxillofac Radiol. 2013;42(10):20130236.

13. Eley KA, Watt-Smith SR, Golding SJ. Three dimensional reconstruction of the craniofacial skeleton with gradient echo magnetic resonance imaging ("Black Bone”): what is currently possible? J Craniofac Surg 2017; 28(2): 463-467

14. Eley KA, Watt-Smith SR, Golding SJ. "Black Bone" MRI: a novel imaging technique for 3D printing. Dentomaxillofac Radiol 2017; 46(3): 20160407

15. Orman G, Wagner MW, Seeburg D, Zamora CA, Oshmyansky A, Tekes A, et al. 
Pediatric skull fracture diagnosis: should 3D CT reconstructions be added as routine imaging? J Neurosurg Pediatr 2015 Oct;16(4):426-431

16. Dremmen MHG, Wagner MW, Bosemani T, Tekes A, Agostino D, Day E et al. Does the Addition of a "Black Bone" Sequence to a Fast Multisequence Trauma MR Protocol Allow MRI to Replace CT after Traumatic Brain Injury in Children? AJNR Am J Neuroradiol. 2017 Nov;38(11):2187-2192.

17. Fleiss JL. Measuring nominal scale agreement among many raters. Psychol Bull $1971 ; 76: 378-82$

18. Brenner D, Elliston C, Hall E, Berdon W. Estimated risks of radiation-induced fatal cancer from pediatric CT. AJR Am J Roentgenol 2001 Feb;176(2):289-96

19. Ida M, Wakayama T, Nielsen ML, Abe T, Grodzki DM. Quiet T1-weighted imaging using PETRA: initial clinical evaluation in intracranial tumor patients. J Magn Reson Imaging $2015 \mathrm{Feb} ; 41(2): 447-453$

20. Serai SD, Laor T, Dwek JR, Zbojniewicz AM, Carl M. Feasibility of ultrashort TE (UTE) imaging of children at 1.5 T. Pediatr Radiol 2014 Jan;44(1):103-8.

21. Greenes DS, Schutzman SA. Clinical significance of scalp abnormalities in asymptomatic head-injured infants. Pediatr Emerg Care 2001 Apr;17(2):88 -92

22. Dunning J, Daly JP, Lomas JP, Lecky F, Batchelor J, Mackway-Jones K, et al. Derivation of the children's head injury algorithm for the prediction of important clinical events decision rule for head injury in children. Arch Dis Child 2006 Nov;91(11):885-91

23. Schutzman SA, Greenes DS. Pediatric minor head trauma. Ann Emerg Med 2001 Jan;37(1):65-74

24. Kralik SF, Yasrebi M, Supakul N, Lin C, Netter LG, Hicks RA, et al. Diagnostic 
performance of ultrafast brain MRI for evaluation of abusive head trauma. AJNR Am J Neuroradiol 2017 Apr;38(4):807-813 
Table 1. Diagnostic performance of Black Bone MRI compared to gold standard Head CT for detection of skull fracture.

\begin{tabular}{lccccc} 
& \multicolumn{2}{c}{ MRI Strength } & \multicolumn{2}{c}{ Sedation } \\
& $\begin{array}{l}\text { All Patients } \\
(\mathbf{n}=\mathbf{3 4})\end{array}$ & $\mathbf{1 . 5} \mathbf{T}(\mathbf{n}=\mathbf{1 1})$ & $\mathbf{3 T}(\mathbf{n = 2 3 )}$ & No sedation (n=19) & Sedation (n=15) \\
\hline Sensitivity & $83 \%$ & $100 \%$ & $75 \%$ & $50 \%$ & $100 \%$ \\
Specificity & $100 \%$ & $100 \%$ & $100 \%$ & $100 \%$ & $100 \%$ \\
PPV & $100 \%$ & $100 \%$ & $100 \%$ & $100 \%$ & $100 \%$ \\
NPV & $97 \%$ & $100 \%$ & $95 \%$ & $94 \%$ & $100 \%$ \\
Accuracy & $97 \%$ & $100 \%$ & $96 \%$ & $95 \%$ & $100 \%$
\end{tabular}


Figure 1. An 18-month-old female with multiple skull fractures (arrows) demonstrated on axial head $\mathrm{CT}$ and 3D CT reconstruction $(\mathrm{A}, \mathrm{C})$ and axial black bone MRI and 3D MRI reconstruction $(\mathrm{B}, \mathrm{D})$.

Figure 2. A 9-month-old female with multiple skull fractures (arrows) demonstrated on coronal head CT and 3D CT reconstruction $(\mathrm{A}, \mathrm{C})$ and coronal black bone MRI and 3D MRI reconstruction $(\mathrm{B}, \mathrm{D})$.

Figure 3. A 4-month-old female with isolated right parietal skull fracture (arrow) demonstrated on axial head CT and 3D CT reconstruction $(A, C)$ but not identified on axial black bone MRI or 3D MRI reconstruction (B,D). 


\title{
Black Bone MRI with 3D reconstruction for the Detection of Skull Fractures in Children with Suspected Abusive Head Trauma
}

\author{
Stephen F. Kralik, MD ${ }^{1}$ \\ steve.kralik@gmail.com \\ Nucharin Supakul, MD² \\ tsupakul@iupui.edu \\ Isaac C. $\mathrm{Wu}, \mathrm{MD}^{2}$ \\ icwu@iupui.edu \\ Gaspar Delso, $\mathrm{PhD}^{3}$ \\ gd410@cam.ac.uk \\ Rupa Radhakrishnan, $\mathrm{MD}^{2}$ \\ rradhak@iu.edu \\ Chang Y. Ho, $\mathrm{MD}^{2}$ \\ cyho@iupui.edu \\ Karen A Eley, FRCR, DPhil, MD ${ }^{3}$ \\ Karen.a.eley@gmail.com \\ ${ }^{1}$ Department of Radiology \\ Texas Childrens Hospital \\ 6701 Fannin St, Suite 470 \\ Houston, TX, 77030 \\ Tel:1 (832) 822-0406 \\ Fax: 1 (832) 825-0300 \\ ${ }^{2}$ Department of Radiology and Imaging Sciences, \\ Indiana University School of Medicine \\ 714 N. Senate Avenue \\ Indianapolis, Indiana \\ Tel:1 (317) 963-7121 \\ Fax: 1 (317) 948-8383 \\ ${ }^{3}$ Department of Radiology, \\ University of Cambridge, \\ Addenbrooke's Hospital, \\ Hills Road, \\ Cambridge CB2 0QQ, \\ United Kingdom
}


Disclosure/Conflict of interests: G.D, is employed by GE Healthcare.

Funding: No source of funding was used for this research.

\section{Ethical Approval:}

This research was approved by the Institutional Review Board of Indiana University.

All procedures performed in studies involving human participants were in accordance with the ethical standards of the institutional and/or national research committee and with the 1964 Helsinki declaration and its later amendments or comparable ethical standards.

For this type of study formal consent is not required.

Correspondence to:

Stephen F. Kralik M.D.

Department of Radiology

Texas Children's Hospital

6701 Fannin St, Suite 470

Houston, TX, 77030

Tel:1 (832) 822-0406

Fax: 1 (832) 825-0300

E-mail: steve.kralik@gmail.com 


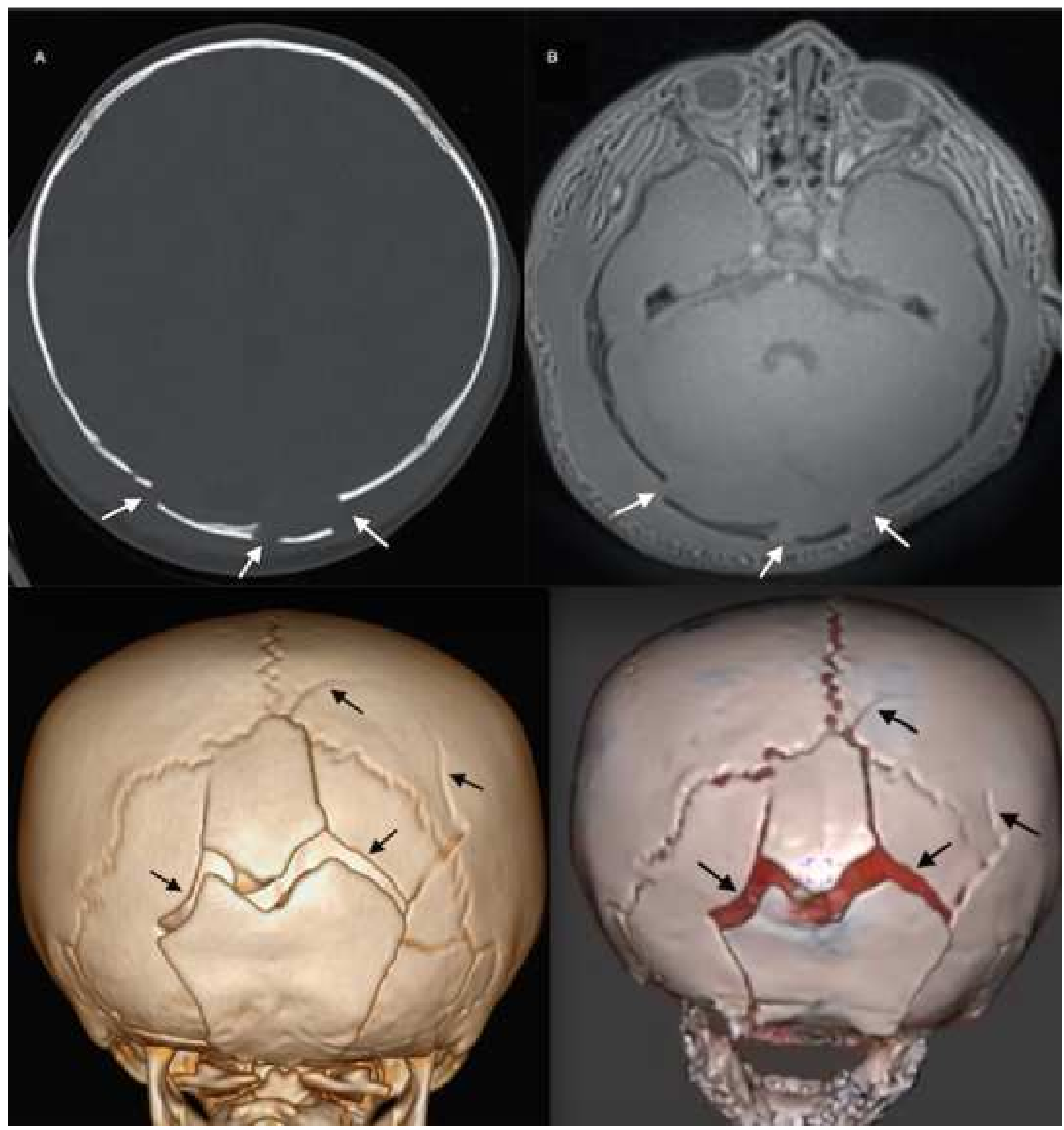


Click here to access/download;Colour Figure;Figure 2.png $\underline{\underline{ \pm}}$

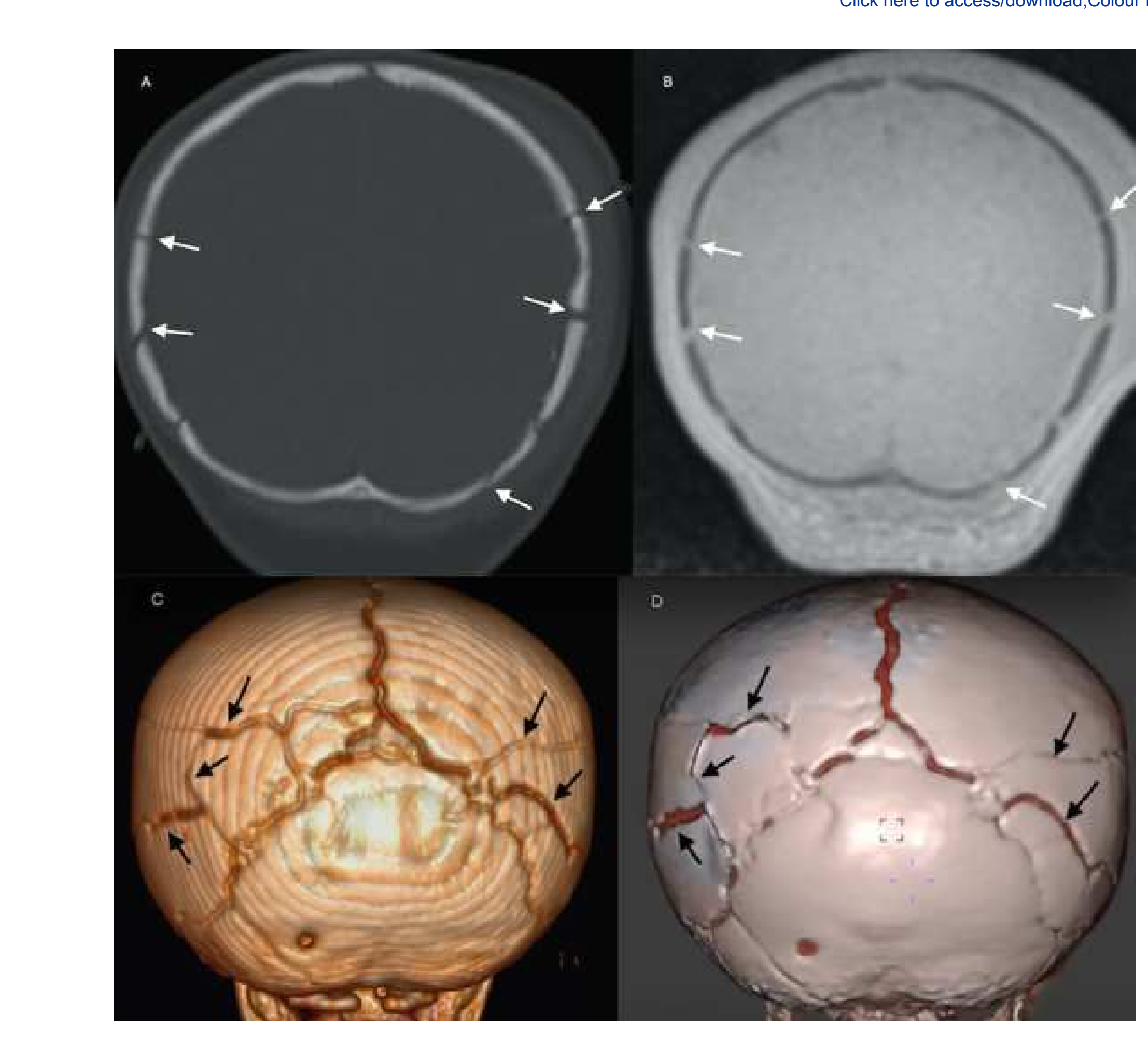


Click here to access/download;Colour Figure;Figure 3.png $\underline{\underline{ \pm}}$

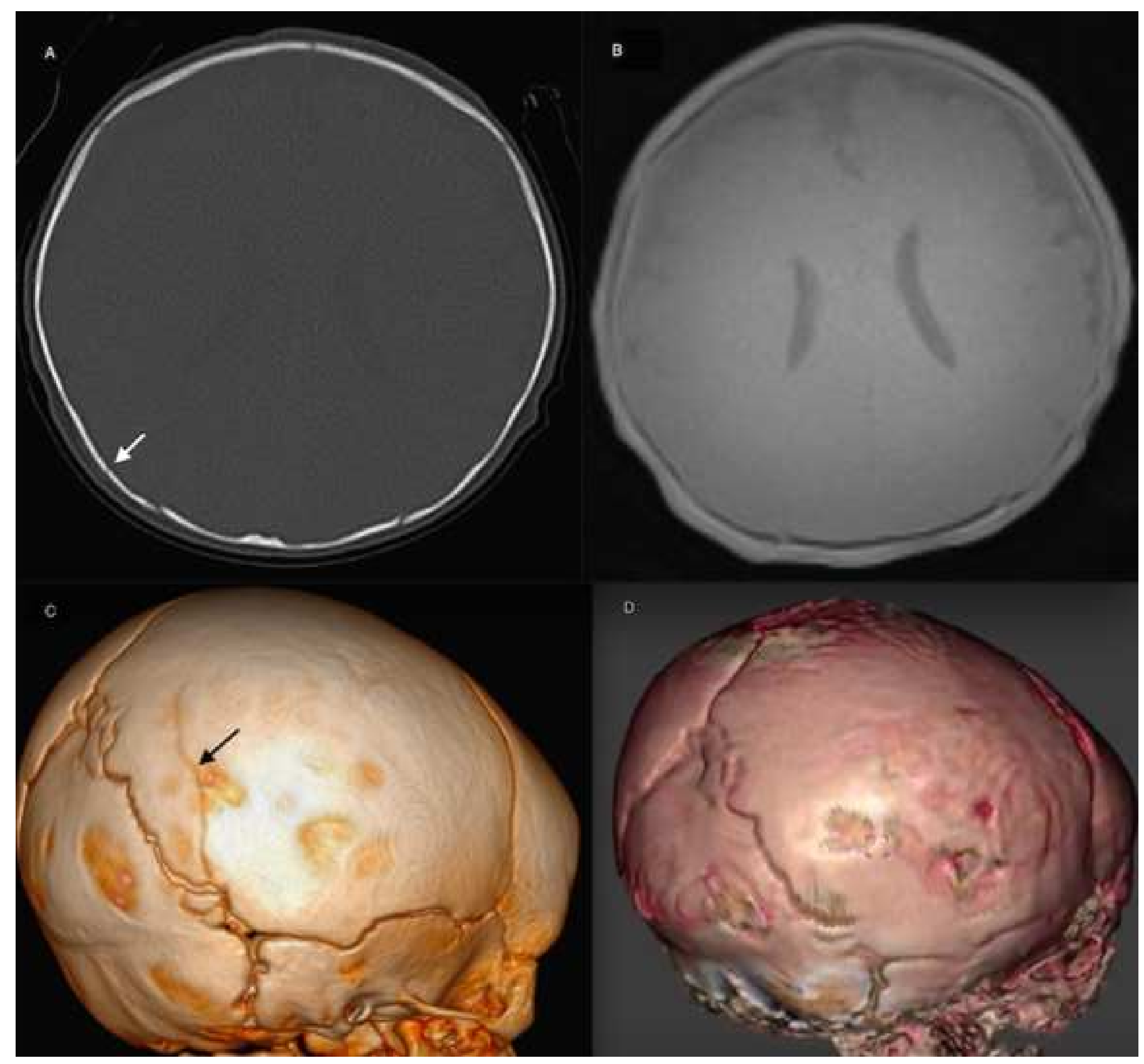

